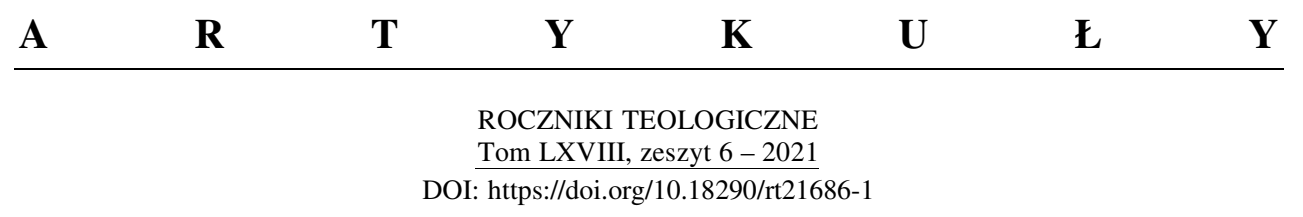

RICHARD HARTMANN

\title{
AUF DER SUCHE NACH DER WAHRHEIT GOTTES - NEUES SELBSTVERSTÄNDNIS DER KIRCHE
}

\author{
IN SEARCH OF THE TRUTH OF GOD \\ - A NEW SELF-UNDERSTANDING OF THE CHURCH
}

\begin{abstract}
After the great loss of credibility in her authority, the structure of the Catholic Church is in the central process of transformation. The Synodal Path in Germany is an attempt to allow others to recognize the transition from an objectivist to a communicative understanding of the truth. The process is not primarily about concrete reform proposals that are acceptable to the majority. Rather, the questions concerning both the unity of faith and the hermeneutic processes of formulating faith are in the center of attention.
\end{abstract}

Keywords: truth; synodality; alterity; credibility; enlightenment; secularization; authority; episcopate.

Wahrheit ist zu einem ideologischen Kampfbegriff geworden. Dies zeichnet die Kommunikationsprobleme des Synodalen Weges der Kirche in Deutschland aus. Es ist jedoch ein Phänomen, das sich in den Polarisierungen etlicher Gesellschaften zeigt. Mit Kopfschütteln wird dies wahrgenommen von einer Gesellschaft, deren Funktionieren auf der Basis des Kompromisses beruht. Wenn dann in den USA ein Präsident ohne große Folgen für seine politische Karriere zigtausende Fake-News twittern kann und dennoch Millionen Stimmen hinzugewinnt, macht das unruhig. „Fake oder Fakt“ war ein Motto der digitalen Familientagung eines katholischen AkademikerVerbandes: Heiligenstadt - ND Christsein. Heute (nd-netz.de), der sich dem

Prof. Dr. Richard HARTMAnN - Lehrstuhl für Pastoraltheologie und Homiletik an der Theologischen Fakultät Fulda, Priester der Diözese Mainz; e-mail: Hartmann@thf-fulda.de; ORCID: https://orcid.org/0000-0003-4838-6347. 
Thema zwischen den Jahren 2020/21 stellte ${ }^{1}$. Was prägt uns und wie finden wir als Christinnen und Christen Orientierung?

\section{DIE DEFINITIONSMACHT DER WAHRHEIT DES GLAUBENS IST GESCHWUNDEN}

Was noch bis in die 50er Jahre für einen großen Teil der Katholiken selbstverständlich war, dass nämlich in Sachen des Glaubens der Hierarchie zu gehorchen sei, wird nur noch von kleinen Teilen der Kirche heute akzeptiert. Das Ansehen der Autorität insgesamt und der kirchlichen Autorität im Besonderen ist weitgehend zerbrochen. Formulierungen wie die erste Regel über die kirchliche Gesinnung im Exerzitienbuch des Ignatius von Loyola stoßen auf Widerstand: „Jegliches (eigenmächtiges) Urteilen zurücksetzend, müssen wir den Geist bereit und willig halten, um in allem der wahren Braut Christi unseres Herrn zu gehorchen, die da ist unsere heilige Mutter, die hierarchische Kirche“2. Metaphern von der heiligen Mutter, der hierarchischen Kirche werden von kaum jemanden noch akzeptiert. P. Peter Knauer SJ ordnet diese Regeln, speziell die 13. Regel, später so ein: „Die 13. Regel lautet: ,Wir müssen immer festhalten, um in allem das Rechte zu treffen: Von dem Weißen, das ich sehe, glauben, dass es schwarz ist, wenn die hierarchische Kirche es so bestimmt, indem wir glauben, dass zwischen Christus unserem Herrn, dem Bräutigam, und der Kirche, seiner Braut, der gleiche Geist ist, der uns leitet und lenkt zum Heil unserer Seelen. Denn durch den gleichen Geist und unseren Herrn, der die Zehn Gebote gegeben hat, wird gelenkt und geleitet unsere heilige Mutter Kirche' (GÜ 365).

Wenn man nicht so genau hinschaut, scheint dies zu besagen, dass die Kirche immer Recht hat, mag sie verkündigen, was immer sie will. Manche Übersetzungen schreiben dann auch gleich: ,Von dem, was mir weiß scheint, glauben, dass es schwarz ist'. Der Vernunfteindruck kann nur ein Schein gewesen sein. Tatsächlich gibt sich ja die Vernunft oft und gerne mit dem Schein zufrieden; aber das gilt leider auch von einer solchen Textlektüre.

Liest man die Regel - vernünftigerweise! - etwas aufmerksamer, dann fällt erstens auf, dass nirgends von bloßem Schein die Rede ist. Es heißt , das

\footnotetext{
${ }^{1}$ Manche Aspekte verdanke ich dem Beitrag von Prof. Dr. Ute Leimgruber, Regensburg, bei jener Tagung.

${ }^{2}$ Ignatius von Loyola, Geistliche Übungen: Übertragung aus dem spanischen Urtext, Erklärung der 20 Anweisungen von Adolf Haas (Freiburg: Herder, 1967), Nr. 353.
} 
Weiße, das ich sehe (lo blanco que yo veo)‘. Es geht um wirkliches Sehen und ausdrücklich um das eigene Sehen und nicht etwa nur eine herrschende Meinung (im Spanischen ist das yo immer betont). Zweitens soll eine allgemeine Regel nicht nur für Randfälle, sondern für überhaupt alle Glaubensaussagen aufgestellt werden; sie würden sonst völlig missverstanden. Und drittens ist der eigentliche Grund für die Regel der, dass es in Glaubensaussagen um eine Erkenntnis im Heiligen Geist geht. Der Heilige Geist ist ein und derselbe in Christus und uns. Wir werden vom Heiligen Geist zu dem hingeleitet, was unser eigenes ewiges Heil ist.

Tatsächlich handeln alle Glaubensaussagen von der Verbindung von etwas Geglaubtem mit etwas Gesehenem. Im Glauben geht es um Gottes Selbstmitteilung an sein Geschöpf. Deshalb haben Glaubensaussagen immer die Struktur einer Einheit von Gegensätzen. Ich sehe den Gekreuzigten, einen zu Tode geschundenen Menschen. An dieser Sicht ist nichts unzutreffend; sie ist alles andere als bloßer Schein. Aber ich glaube an ihn als den Auferstandenen. Seine Gottessohnschaft angesichts des Todes ist seine Auferstehung. Dies ist keiner anderen Erkenntnis zugänglich als demjenigen Glauben, der das Erfülltsein vom Heiligen Geist ist. «Niemand kann sagen: ,Jesus ist Herr', außer im Heiligen Geist.» (1 Kor 12,3).

Ähnliches gilt von der Kirche. Wir sehen eine Gemeinschaft von Menschen mit vielen Fehlern. Das ist kein Schein, sondern die volle Wahrheit. Aber wir glauben, dass Gott uns in der Glaubensverkündigung der Kirche seine eigene Gegenwart schenkt. Wir glauben immer Gegensätzliches zu dem, was wir sehen, ohne Letzteres dementieren zu müssen“33. Eine banale In-Eins-Setzung von Bischöflicher Lehrentscheidung und gläubiger Annahme durch die Christen ohne die Gemeinschaft beider im Heiligen Geist ist also unzulässig.

Kirchliche Autorität steht somit schon in dieser Sicht in Spannung zur Verstandes- und Gewissensentscheidung des Einzelnen.

Mit Charles Taylor beginnen diese Veränderungen, die die Anerkennung der jeweiligen Autoritäten ${ }^{4}$ relativieren im 17. Jahrhundert: „So kam es, dass

\footnotetext{
${ }^{3}$ Peter Knauer, Den Verstand abgeben? Ignatius von Loyola über Sehen und glauben (2010). Accessed: December 31, 2020, http://peter-knauer.de/ignatius1.html.

${ }^{4}$ Eigens müsste man darüber reflektieren, wie die Lehrbekenntnisse in der frühen Kirche in ihrer Verbindlichkeit sich entwickelt haben. Edmund Arens weist in seiner Untersuchung (Edmund Arens, Bezeugen und Bekennen elementare Handlungen des Glaubens (Düsseldorf: Patmos, 1989) darauf hin, dass das Nizänum nicht als rein kirchlich-theologisches Projekt verstanden werden kann. Das Interesse „,des nunmehr christlichen Kaisers Konstantin [...], auf einer durch ihn einberufenen kaiserlichen Reichssynode seine auf die Einheit abzielenden reichspolitischen Absichten auch mit Hilfe einer Einheitsformel durchzusetzen“ (ebd., 265), kommt zum Tragen. Einheitsformulierungen
} 
sich der ausgrenzende Humanismus durchsetzen konnte und zwar nicht bloß als eine von einer winzigen Minderheit verfochtene Theorie, sondern als eine immer tragfähigere spirituelle Auffassung. Damit sie zum Vorschein kommen konnte, mussten zwei Bedingungen erfüllt sein: erstens die negative Bedingung des Verschwindens der verzauberten Welt und zweitens die positive Bedingung, dass eine tragfähige Konzeption unserer höchsten spirituellen und moralischen Bestrebungen derart zustande kommt, dass wir uns vorstellen können, diese Ziele anzuerkennen und zu verfolgen, ohne dass Gott ins Spiel kommt ${ }^{* 5}$. Das Verstehen der Welt ist nicht mehr abhängig von der Anerkennung Gottes oder einer von ihm legitimierten Autorität.

Die Aufklärung im 18. Jahrhunderts stürzte die ,gottgegebenen“ Autoritäten vom Thron und führte zu einer ausdrücklichen Orientierung an der Vernunft. Mit Friedrich Schleiermacher ist „Aufklärung im anspruchsvollen Sinne [...] eben keine Epoche der Geistesgeschichte, sondern eine bleibende Aufgabe, der sich weder die Religion, noch andere Bereiche des Lebens entziehen sollen. [...] Mündigkeit versetzt in die Lage, kundig Auskunft zu geben, denn Mündigkeit kommt von Mündlichkeit, sie steht für das Sprachvermögen, für das individuelle Gewissen, für die kritische Urteilskraft des Einzelnen. Keine Autorität der Welt kann diese Mündigkeit beschneiden oder gar verbieten, ohne Verrat an den Wurzeln des christlichen Glaubens zu begehen. Mündigkeit und Sprachvermögen nötigen zwangsläufig zur Bildung in und über die eigene Religion. Mündigkeit ist demnach weniger ein Zustand als eine Übung. Mündigkeit ist auch die erste Voraussetzung für einen Dialog der Religionen, der seinen Namen verdient. Denn nur wer mündig spricht, der hat eine Position, von der aus er mit Haut und Haaren argumentiert, ohne beim ersten Angriff sofort umzufallen“ “6. Solche Mündigkeit nehmen die Menschen des 21. Jahrhunderts für sich ausdrücklich in Anspruch und üben sie im gesellschaftlich demokratischen Dialog.

Im 19. Jahrhundert bäumten sich die bisherigen Autoritäten noch einmal gegen diesen Prozess auf. Kirchlich ist dies besonders im Pontifikat Pius IX.

erweisen sich daher als politische Strategien und sind hier nicht primär einem Wahrheitsanspruch folgend, der von kirchlichen Autoritäten symbolisiert wird.

${ }^{5}$ Charles Taylor, Ein säkulares Zeitalter (Frankfurt a. M.: Suhrkamp, 2009), 401 f., zitiert nach Jan Loffeld, Der nicht notwendige Gott: Die Erlösungsdimension als Krise und Kairos des Christentums inmitten seines säkularen Relevanzverlustes (Würzburg: Echter Verlag, 2020), 122.

${ }^{6}$ Petra Bahr, ,Religion und Aufklärung“, Rheinischen Merkur, 9. März 2006. Accessed: Januar 4, 2021, https://www.ekd.de/060313_bahr_religion_aufklaerung.htm. 
zu verfolgen. Noch einmal wurde versucht mit allen Mitteln kirchlicher Verabsolutierung die hierarchische Unfehlbarkeit zu divinisieren ${ }^{7}$.

Im europäischen Kulturraum waren es dann vor allem der Holocaust und die Diktaturen des Nationalsozialismus und später des „realen Sozialismus“ der DDR, die ikn der folgenden Reflexion Autorität grundsätzlich unter Verdacht stellten. Was sich in den Studentenunruhen der 68er Bahn brach, war die Erkenntnis, dass sowohl politische wie kirchliche Obrigkeiten ${ }^{8}$ ihre Fehleinschätzungen und ihre Verstrickungen in verheerende Fehlurteile nicht mehr verbergen konnten. Je mehr sie sich selbst als nicht kritisierbare Autoritäten konstruierten, desto mehr wurde ihr Autoritätsanspruch hinterfragt und meistens erfolgreich dekonstruiert. Wer mit Autorität auftrat, konnte diese nicht mehr in einem Machtanspruch, potestas durchsetzen, sondern höchstens in einer persönlichen Art der Glaubwürdigkeit als auctoritas erwerben.

Als immer stärkeres Kriterium für die Anerkennung als richtig und falsch, entwickelten sich zeitweise die dominierenden Wissenschaftspositionen. Als wahr galt, was in den Naturwissenschaften in einer positivistischen Manier „bewiesen“ war. Als eichtig galt, was in ökonomischer Praxis erfolgreich war. Die Veränderungen der Leitwissenschaften der Universitäten folgten dieser Verschiebung. Betriebs- und Volkswirtschaftslehre und die MINT-Fächer (die Bereiche Mathematik, Informatik, Naturwissenschaft und Technik) dominierten die Wissensgesellschaft.

Parallel zu solchen wissenschaftsgläubigen Positionen, entwickelte sich eine neue Elite der medial sich selbst präsentierenden Persönlichkeiten, mediale Autoritäten, wie sie am einflussreichsten in den sozialen Medien als Influencer Einfluss gewinnen. Dies verstärkt sich, wenn sich im medialen Overload von Informationen zur Bewältigung der Komplexität eine neue Ausprägung von Versäulung, von begrenzten, zum Teil durch Algorithmen gesteuerten digitale Netzen mit ihren kommunikativen „Blasen“ gegenseitig bestätigten. Auch diese Entwicklung spiegelt sich in kirchlich geprägten Umfeldern.

Eine neue Form der Konfessionalisierung entwickelt sich. Was lange Zeit einfach geprägt wurde durch die Auswahl der verschiedenen Printmedien (,Wenn du mir sagst, was du liest, sage ich dir, wie du tickst!“) verstärkt sich dies mehr und mehr in Spartenkanälen und Webseiten sowie entsprechenden Blogs und anderen social networks, die ihr Profil nicht zuletzt in der Abgrenzung

\footnotetext{
${ }^{7}$ In jüngerer Zeit werden immer mehr historische Zusammenhänge aufgedeckt. Instruktiv ist dazu u. a. Peter Neuner, Der lange Schatten des I. Vatikanums wie das Konzil die Kirche noch heute blockiert (Freiburg Basel Wien: Herder, 2019) und Hubert Wolf, Der Unfehlbare Pius IX. und die Erfindung des Katholizismus im 19. Jahrhundert: Biographie (München: C.H. Beck, 2020).

${ }^{8}$ Hier sei ausdrücklich auf die widersprüchlichen Positionen der Bischöfe im III. Reich verwiesen.
} 
von anderen Positionen gewinnen. Echte Kommunikation miteinander, Kommunikation jenseits der eigenen Meinung und Einschätzung wird immer seltener.

Spätestens in den 60er Jahren nahm der Prozess noch mehr an Fahrt auf. Nicht mehr aufgrund der formalen Autoritätszuschreibungen, sondern aufgrund individueller und biographischer Aspekte oder ihrer Selbstinszenierung gewannen die Autoritäten Glaubwürdigkeit.

Die gesellschaftlichen Veränderungsprozesse zeigen sich in gleicher Weise in der kirchlichen Übernahme. Das programmatische Wort von Niklas Luhmann über die „Religion der Gesellschaft“" ${ }^{\text {“9 }}$ eigt sich auch darin, dass die Kirche immer die Kirche der Gesellschaft ist, so sehr sie sich auch als Gegenbild inszenieren will.

Hierarchisches Amt und der Glaube an die damit verbundene sakramentale Gnade werden nicht mehr als tragfähige Begründungsfiguren akzeptiert ${ }^{10}$. Die durch die Mediengesellschaft geförderte Präsentation der Symbolfiguren einer Organisation oder Institution führen zwar dazu, dass mehr und mehr die Bischöfe als Platzhalter für Kirche, aber zugleich als Platzhalter für Glaube und Theologie fungierten. Zugleich wurden sie in ihrer Glaubwürdigkeit auch immer stärker angefragt. Die Bewertung der von ihnen vertretenen Inhalte wird nicht selten durch die Rollenidentifikation verdeckt. Etliche kirchlich Engagierten klagen darüber, dass sie mit Verhaltensweisen „,der Kirche und ihrer Amtsträger" konfrontiert werden, von denen sie sich selbst doch distanzieren.

Was der gesellschaftlichen Situation geschuldet war, führte im weltkirchlichen Rahmen zur Ausprägung neuer kommunikativer, konziliarer und synodaler Prozesse. Das Vaticanum II, das für etliche Kuriale, die ihren eigenen Verlust an Einfluss erahnten, schon in der Ankündigung ein Schreckgespenst

\footnotetext{
${ }^{9}$ Niklas Luhmann, Die Religion der Gesellschaft (Frankfurt am Main: Suhrkamp, 2000).

${ }^{10}$ Insgesamt scheint mir gerade auch während der Corona-Pandemie des Jahres 2020 immer deutlicher, wie sich die sakramentale Gestaltung der Kirche relativierte. Wenn auch den Katholikinnen und Katholiken die Nutzung medialer und webbasierter Gottesdienstformen empfohlen wird, so zeigt sich immer mehr, das inhaltliche und ästhetische Qualität dieser Produkte wichtiger sind als die jeweilige konfessionelle Ausrichtung. Die Feier der Kommunion, die Sehnsucht nach dem Herrenmahl ist nicht nur für die jeweiligen Kommunionkinder, sondern auch für bislang regelmäßig kommunizierenden Katholikinnen und Katholiken weniger wichtig. Schon länger sinkt die Praxis der Babytaufe, werden kirchliche Trauung und sogar christliches Begräbnis weniger nachgefragt. Im Krankheitsfall kommt es mehr auf den Segen der Kranken an und die Begleitung der trauernden Angehörigen als auf die Feier der Krankensalbung. Und auch in der Begegnung mit hauptberuflich Verantwortlichen der Kirche wird mehr und mehr die erlebbare Kompetenz wertgeschätzt. Ob die Menschen zum ordinierten Amt gehören ist zweitrangig. Die stetige Betonung der Sakramentalität der Kirche und der Feier der sieben Sakramenten verliert m. E. in großer Beschleunigung an Überzeugungskraft.
} 
war, zeigte sich als Prozess, der echte denkerische Fortschritte nach sich zog und bisherige Machteliten schwächte. Ähnliches geschah in der Würzburger Synode (1971-1975), wobei beide kirchlichen Prozesse noch geprägt waren durch das Ziel, in den Ergebnissen möglichst viel Einmütigkeit zu zeigen. Doch beide Prozesse stießen an ihre Grenzen. Symbolisch wirkte die Beratungsresistenz von Papst Paul VI. in Sachen Sexualmoral, für die Kirche in Deutschland die Erfolglosigkeit der Synodal-Voten, die beim Heiligen Stuhl vorgelegt wurden, aber auch nicht mehr nachhaltig dort eingefordert wurden. Der Versuch, statt der in Frage stehenden hierarchischen Autoritäten auf synodale Positionen zu setzen, scheiterte in vielen Fällen am innerkirchlichen Widerstand oder am Rückzug einzelner Bischöfe auf ihre kanonistisch gesicherte Verantwortung.

Diese Erfahrung war jedoch nur der Anfang des Zerbröselns von Autorität. Drei weitere Phänomene beschleunigten weitere Verfallsprozesse.

1. Trotz der „kommunikativen Wende“ entfalteten manche Bischöfe neue Formen eines absolutistischen Klerikalismus und versuchten, sich selbst unangreifbar zu machen. Dies scheiterte jedoch mit großem Flurschaden in etlichen Diözesen. Selbst zerstörend wirkte, wie kirchliche Verantwortliche in Wien (Kardinal Groer), St. Pölten (Bischof Krenn), Chur (Bischöfe Haas und Huonder, aber auch Vorkommnisse wie die verweigerte Neuwahl eines Bischofs aus der römischen Dreierliste durch das Domkapitel 2020), Augsburg (Bischof Mixa) oder Limburg (Bischof Tebartz van Elst) und jüngst im Agieren um die Berichte über den Umgang mit sexuellem Missbrauch im Erzbistum Köln (Kardinal Woelki) in unterschiedlicher Weise die kirchliche Autorität unglaubwürdig machten. Das Verhalten dieser Bischöfe und mit ihnen der gesamten kirchlichen Strukturen waren für die Öffentlichkeit nicht mehr nachzuvollziehen. Auch Katholikinnen und Katholiken aus dem innersten Kern der Kirche gingen auf Distanz. Die Zahl der Kirchenaustritte wuchs in vorher kaum vorstellbare Höhen. Diese Distanzierungen von der kirchlichen Struktur und ihrer institutionellen Ausprägungen waren jedoch kaum gleich zu setzen mit dem Verlust des Glaubens an das Evangelium Jesu Christi. Vielmehr betonten etliche, dass der Institution der Glaube verloren gegangen sei.

Interessanter Weise behalten etliche die Bindung an die Kirche und die Feier des Kirchenjahres und der Sakramente zu Lebenswende trotzdem bei. 
Grace Davie hat dazu die Spannung believing without belonging ${ }^{11}$ herausgearbeitet. Menschen bleiben dem Glauben treu, auch wenn sie vielleicht sogar um des Glaubens willen zur Institution nicht [mehr] dazugehören wollen. An bestimmten Themenfeldern wurden die Brüche besonders deutlich. Mit der Enzyklika Humanae Vitae war bereits das Auseinanderfallen kirchliche Lehre und christlicher Praxis und ihrer Deutung offenkundig geworden. Immer wieder sind es Themenfelder, die die Sexualität betreffen, die nicht mehr von den Gläubigen rezipiert werden: Der Umgang mit wiederverheirateten Geschiedenen, die Bewertung der Homosexualität (verbunden mit dem Aufdecken homosexueller Netzwerke im inneren kirchlichen Bereich), die Diskussionen um das soziale (Gender) und biologisch (Sex) Geschlecht und dann speziell innerkirchlich die Begründungszusammenhänge von priesterlicher Ehelosigkeit und Frauenordination. Nach dem dann mit dem Bekanntwerden des sexuellen und geistlichen Missbrauchs die lange mit Vertrauensvorschuss geschützten Priester und Ordensleute in den Blick der Öffentlichkeit kamen und immer noch kirchlich Verantwortliche eher zu Vertuschungsmaßnahmen statt zu Verantwortungsübernahme neigten, waren die letzten Reste von Glaubwürdigkeit verloren.

2. Der zweite Bereich, der die Krise der Kirche befeuerte, war der Umgang mit den weltlichen Gütern. Seit Jahrzehnten wird von mafiösen Machenschaften im Vatikan berichtet. In Deutschland sind es missbräuchliche Nutzungen (Limburger Bischofshaus) oder riskante, verbrecherische und gescheiterte Finanztransaktionen (Caritas Trier, Bistum Eichstätt und Erzbistum Freiburg, Finanzaktionen des Heiligen Stuhls), die es schwer machen, den kirchlichen Institutionen zu trauen. Dazu kommt, dass die Bedeutung der großen kulturellen Güter der Kirche kaum unterschieden werden können, für die Verfügbarkeit unternehmerischen Handelns. Die Zerfallsprozesse der Kirche sind offenbar unaufhaltsam. Jede kirchliche Autorität unterliegt seither zuerst der Verdachtsvermutung. Die Annahme eines fast „kindlichen“ Vertrauensvorschusses ist kaum jemandem vermittelbar.

3. Die verzweifelte Reaktion der Mehrzahl moderater Bischöfe bei der Frühjahrsvollversammlung der Deutschen Bischofskonferenz in Lingen 2019 war, gemeinsam mit dem Zentralkomitee der Deutschen Katholiken einen „synodalen Weg“ zu beginnen. Vier Krisenfeldern („Macht und Gewaltenteilung in der Kirche - Gemeinsame Teilnahme und Teilhabe am Sendungsauftrag“,

\footnotetext{
${ }^{11}$ Grace Davie, Religion in Britain since 1945: Believing without belonging (Oxford: WileyBlackwell, 1994).
} 
„Priesterliche Existenz heute“ „Frauen in Diensten und Ämtern in der Kirche“, „Leben in gelingenden Beziehungen - Liebe leben in Sexualität und Partnerschaft") wurden identifiziert, um in ihrer Bearbeitung einen neuen Stil vertrauensvollen Miteinanders zu erarbeiten. Bedenken gegen einen solchen „gemeinsamen Weg“ gab es von Anfang an von einer kleinen aber sich deutlich formierenden Gruppe aus kanonistischen und ekklesiologischen Gründen. Zwar wurde, in mancher Anlehnung an die Würzburger Synode, eine Satzung ${ }^{12}$ erarbeitet, die symbolisch sichert, dass gemeinsame Entschlüsse, getragen zugleich mit einer deutlichen Mehrheit der Bischofskonferenz, gefasst werden. Eine Rechtsverbindlichkeit kann vor dem Hintergrund des CIC von 1983 jedoch nicht gewährt werden:

„Artikel 11 Beschlussfassung

(1) Die Synodalversammlung des Synodalen Weges fasst zur abschließenden Feststellung der Beratungsergebnisse Beschlüsse. Sie ist beschlussfähig bei Anwesenheit von wenigstens zwei Dritteln ihrer Mitglieder.

(2) Ihre Beschlüsse erfordern eine Zweidrittelmehrheit der anwesenden Mitglieder, die eine Zweidrittelmehrheit der anwesenden Mitglieder der Deutschen Bischofskonferenz enthält.

(3) Auf Antrag von einem Viertel der anwesenden Mitglieder werden zu den in Schlussabstimmungen gefassten Beschlüssen Sondervoten veröffentlicht.

(4) Grundsätzlich erfolgen Abstimmungen öffentlich. Davon ausgenommen sind Personalentscheidungen sowie Abstimmungen, die auf Antrag von mindestens fünf Mitgliedern der Synodalversammlung geheim erfolgen können.

(5) Beschlüsse der Synodalversammlung entfalten von sich aus keine Rechtswirkung. Die Vollmacht der Bischofskonferenz und der einzelnen Diözesanbischöfe, im Rahmen ihrer jeweiligen Zuständigkeit Rechtsnormen zu erlassen und ihr Lehramt auszuüben, bleibt durch die Beschlüsse unberührt" ${ }^{\text {“13. }}$.

Etliche Symbole wurden gesetzt, die dazu beitragen wollen, die Grundhaltung der Gemeinsamkeit zu unterstreichen. Der Eröffnungsgottesdienst war ausdrücklich als gemeinsame Feier in der Verantwortung von Laien, Religiosen und Klerikern gestaltet. Ungewöhnlich, vor allem wenn man die Bilder der Bischofskonferenz vor Augen hat, dass etliche Bischöfe einfach gemeinsame Plätze mit dem ganzen Gottesvolk haben. Auch die Sitzordnung nimmt nicht auf Rang und Position Rücksicht, sondern nur auf das namentliche Alphabet. Dies alles, wie auch der ausdrückliche Verzicht einiger, ihre

\footnotetext{
${ }^{12}$ Satzung des Synodalen Weges. Accessed: Januar 4, 2021, https://www.synodalerweg.de/fi leadmin/Synodalerweg/Dokumente_Reden_Beitraege/Satzung-des-Synodalen-Weges.pdf.

${ }^{13}$ Ebd.
} 
Amtsautorität gegen andere auszuspielen, verhindert jedoch nicht, dass eine klare Minderheit gerade die Gemeinsamkeit dieses Weges nur mit größten Vorbehalten mitgehen will.

Einer solchen symbolischen Partnerschaft steht eine unterschiedliche Definitionsmacht gegenüber, wie sie vor allem von fundamentalistisch-dogmatistisch geschlossenen Theologiesystemen (Ottmar Fuchs) postuliert wird. So unterstreichen ihre Vertreter: „Wir als Bischöfe haben die sakramentale Verantwortung, die sich keinen Mehrheiten beugen darf“. Dem strukturellen Versuch, Konsens in breiter Mehrheit zu organisieren, steht die machtdominierte Blockade-Politik Einzelner gegenüber.

Der „einfache“ Weg, dem zu begegnen, wäre, diese Positionen und mit ihnen die entsprechenden Personen, auch Bischöfe konsequent auszugrenzen und ihre Positionen inhaltlich zu disqualifizieren. Solche Praxis würde diese in ihrer Vorvermutung bestärken, hier werde eine andere, eine neue deutsche Kirche konstruiert, die sich von der Tradition der Weltkirche, des Heiligen Stuhles und des sakramental-hierarchisch verfassten Bischofsamtes absetzen. So wissen sie sich ins Martyrium gedrängt und können nur noch auf die kleine Schar der Aufrechten hoffen. Doch stehen dahinter wesentlich zentralere theologische Fragen.

\section{WER BESITZT DIE WAHRHEIT?}

Es lohnt, mit einer hermeneutischen Sensibilität die Heilige Schrift zu durchforschen, um nach dem Zugriff auf Gott und den Zugriff auf die Wahrheit zu fragen. Vor aller exegetischen Detailarbeit ist schon eine Durchsuchung der biblischen Texte nach dem Begriff Wahrheit erhellend. Ich erlaube mir hier solche exemplarischen Assoziationen, um alle anzuregen, hier grundlegender und umfassender den verschiedenen biblischen Deutungen nachzugehen:

1. Gotteserkenntnis ist Gnade und Geschenk von Gott her, sie wird in unterschiedlicher Weise Menschen gewährt, ohne sie damit mit Macht und amtlicher Würde zu bestärken: „Niemand hat Gott je gesehen. Der Einzige, der Gott ist und am Herzen des Vaters ruht, er hat Kunde gebracht“. (Joh 1,18) Der Zugang zu Gott ist an ganz vielen Stellen ein Zugang im Modus der Suche. Gerade auch die Auferstehungsdokumente verdeutliche, dass der Auferstandene sich immer wieder den Jüngern entzieht. Am deutlichsten findet sich dies in der Emmausgeschichte (Lk 24,13-35). 
2. „Wenn ihr in meinem Wort bleibt, seid ihr wahrhaft meine Jünger. Dann werdet ihr die Wahrheit erkennen und die Wahrheit wird euch befreien“. (Joh 8,31 f.) Die Erkenntnis der Wahrheit zeigt sich in der Treue zu Jesus Christus und führt daraus nicht zur Macht, sondern zu Freiheit. Der Zugang zur Macht wird und muss sich daher auch in der Wirksamkeit, in den Früchten $($ Mt 7,16) zeigen.

3. Die Kritik der biblischen Texte stellt immer wieder Autoritäten in Frage (Mt 23,4). Jesus ist vorsichtig gegenüber den herrschenden Schriftgelehrten. Im Blick auf die Jüngergemeinde warnt er vor der Gefahr, sich zu überheben. Ausdrücklich wird dies auch darin sichtbar, dass er solchen Verantwortung und Vertrauen gibt, die selbst immer wieder scheitern. Eine der profiliertesten Personen dabei ist Petrus. Wer versucht, allein aus dem Binde- und Lösewort (Mt 18,18) eine absolute Autorität für die Kirche abzuleiten, verfällt in eine sehr verkürzte Auslegungstradition. Solche Engführung kann ideologiekritisch einfach entlarvt werden.

4. Wichtig ist, innerlich anzunehmen, dass die Beziehung zu Christus als Person selbst erst eine Ahnung der Wahrheit eröffnet, wenn er sich selbst als Weg, Wahrheit und Leben präsentiert (Joh 14,6).

Wahrheit wird sich nicht in menschlichen Zeilen und in amtlicher Autorität festhalten lassen. Sie wird, so scheint mir wichtig festzuhalten zu sein, zuerst im Modus der Suche uns begegnen, uns als Klerikern, als Theologinnen und Theologen und als Gläubige und Suchende an anderen Orten. Nicht die Gewissheit wird uns frei machen, sondern die Frage nach Gott und die Beziehung, die wir zu ihm aufnehmen.

Es gibt seit alters her theologische und philosophische Positionen, die nicht zuletzt deshalb einer reinen Pluralität das Wort reden, den Relativismus gegen die Korrespondenztheorie positionieren und die Wahrheit als solche für nicht existent halten. Den Widerspruch, dass die Aussage „Es gibt keine Wahrheit" selbst am eigenen Wahrheitsanspruch scheitert, wird dann verdrängt.

Für mich ist wichtiger, darauf zu beharren, dass es für uns keine absolute Verfügbarkeit der Wahrheit gibt. Ich muss zugeben, dass die Erkennbarkeit von Wahrheit, die Definierbarkeit ebenso jenseits unserer denkerischen Möglichkeiten liegt, wie die Definierbarkeit Gottes selbst. Das Verhältnis des Menschen zur Wahrheit ist und bleibt somit eine Grundhaltung der Suche. 


\section{ANERKENNUNG DER JE ANDEREN}

In den Diskussionen um den Synodalen Weg in Deutschland wird von den Kontrahenten immer neu kontrovers behauptet, ob die Themen, die verhandelt werden, Grundwahrheiten des Glaubens berühren. Meine Skizze kann helfen, diese Frage insgesamt mit Vorsicht zu diskutieren. Vielmehr können wir vielleicht klären, ob bisherige grundlegende Konsensformulierungen in Frage stehen, wie eventuell die frühkirchlichen Symbola. Auch wäre die Zeitbedingtheit bestimmter dogmatischer Entscheidungen hermeneutisch neu herauszuarbeiten. Schließlich wird es darum gehen, die Pluralität von Zugängen zum Glauben und zur konkreten kirchlichen Praxis anzuerkennen. Diese Grundentscheidungen können die Kirche in ihrer versöhnten Verschiedenheit besser gegenwartsfähig machen als die Absolutsetzung von Positionen der einen wie der anderen Richtung. Auch für die Römische Bischofssynode wird es darauf ankommen, nicht bestimmten Teilen die Rechtgläubigkeit abzusprechen, sondern - vielleicht auch längerhin - die unterschiedliche Praxis zu erdulden. Das alles wird sicher für die Menschen, die von der Sicherheit ihres Zugriffs auf Wahrheit überzeugt sind - im langen Schatten des Pianischen Zeitalters - einiges abverlangen. Aber auch die Gruppierungen, die den genauen Weg der Kirche im 3. Jahrtausend jetzt zu kennen glauben, könnten etwas bescheidener in ihren Erkenntnisansprüchen sein... Letztlich wird die Kirche für sich neu annehmen müssen, als suchende Kirche auf dem Weg zu sein. Wesentlich wird es darauf ankommen, eine Haltung anzunehmen, die darin reift, den anderen anzuerkennen. Paulo Suess entwickelt dazu das Konzept einer „Kultur der Anerkennung““14. „An-Erkennung ist nur möglich im Rückgriff auf Erkennen und Erkenntnis. Erkennen und Verstehen - darum geht es ja bei einer Anerkennungshermeneutik - setzen Erfahrung im Umgang mit den Anderen im Anderssein voraus. [...] Dem erfahrungsarmen, von Vorurteilen und Selbstbespiegelungen bedrohten Erkennen soll [...] Erinnerung zu Hilfe kommen. Erinnerung könnte abstrakter, zeitloser Vernunft die für das Lebensprojekt der Anderen notwendige anthropologische und historische Dimension geben“15. Diese Beobachtungen können m. E. helfen, die Gräben der Kommunikationsabbrüche neu zu überbrücken. Besonders die Erinnerungsarbeit wäre eine zentrale Aufgabe für den Synodalen Weg. In diesem

\footnotetext{
${ }^{14}$ Paolo Suess, „Über die Unfähigkeit der Einen, sich der Andern zu erinnern“, in Anerkennung der Anderen eine theologische Grunddimension interkultureller Kommunikation; [Helmut Peukert zum 60sten Geburtstag], ed. Edmund Arens (Freiburg: Herder,1995), 64-94, hier 64.

${ }^{15}$ Ebd., 65.
} 
Prozess muss auch die Idee der „einen Vernunft“ kritisch reflektiert werden. Suess warnt dafür, dass diese ,ständig über ihre eigene Provinzialität aufgeklärt werden" ${ }^{\text {"16 }}$ muss. Mit Habermas könnte man sagen, dass ihre zugrundeliegenden erkenntnisleitenden Interessen aufgedeckt werden müssen in einem stets diskursiven Verfahren. Edmund Arens ${ }^{17}$ legt in seinen religionstheoretischen Überlegungen „,vier Formen kommunikativ-religiöser Praxis“ ${ }^{18}$ vor, die vielleicht auch helfen können, die kommunikativen Bedingungen des synodalen Weges zu befördern:

\section{„1. Erzählen}

Religiöser Glaube muß erzählt werden; das Erzählen von Glaubensgeschichten ist eine grundlegende kommunikativ-religiöse Praxis. Selbst wo die zentralen Inhalte einer Religion in Glaubensbekenntnissen festgehalten sind, so sind diese doch geronnene ,kondensierte Geschichte', die aus erzählter Geschichte stammen und nach Erzählung ihrer Geschichte verlangen [FN: I.U. Dalferth, Religiöse Rede von Gott, München, 1981, 659]. Religionen reden von Göttern bzw. Gott, indem sie von deren oder dessen Handeln erzählen. Die kosmischen Geschehnisse ,am Anfang', die Schöpfung der Welt und der Menschen, die Urzeit, der Ursprung des Todes sind Gegenstand religiöser Erzählungen, von Schöpfungs- und Urzeitmythen [FN: Zur theologisch notwendigen Mythenhermeneutik und -kritik vgl. I.U. Dalferth, Jenseits von Mythos und Logos. Die christologische Transformation der Theologie (QD 142), Freiburg, 1993], die in der kommunikativ-religiösen Praxis des Erzählens weitererzählt werden wollen“ (ebd., 158). Vermutlich werden die Erzählungen auf dem Synodalen Weg weiterführen als die Versuche von Definitionen. Es wären dann aber auch mögliche auf Zukunft entworfene Erzählungen über die Lebenschancen unter den Augen Gottes.

\section{„2. Feiern}

Eine zweite grundlegende Form kommunikativ-religiöser Praxis ist das Feiern. [FN: Vgl. V. Turner (ebd.), Celebration. Studies in Festivity and Ritual, Washington (DC), 1982; J. Assmann / T. Sundermeier (Hg.), Das Fest und das Heilige. Religiöse Kontrapunkte zur Alltagswelt, Gütersloh, 1991]. In allen Religionen hat der Dienst an den Gottheiten oder der Gottheit den Charakter der Feier. Gottesdienst muß gefeiert werden, und insofern ist Feiern

\footnotetext{
${ }^{16}$ Ebd., 84.

${ }^{17}$ Edmund Arens, „Konturen einer praktischen Religionstheorie“, in Anerkennung der Anderen eine theologische Grunddimension interkultureller Kommunikation, 138-166.

${ }^{18}$ Ebd., 157.
} 
die adäquate Form gottesdienstlichen Handelns“ (ebd., 159). Dies war wohl bei der Eröffnung des Synodalen Weges in Frankfurt zu spüren. Es wäre zu wünschen, dass nach den coronabedingten Beschränkungen diese Dimension wieder ihre Wirkung entfaltet.

\section{„3. Verkündigung}

Eine dritte Grundform kommunikativer-religiöser Praxis ist das Verkündigen. Durch Verkündigen werden die religiösen Inhalte mitgeteilt, damit sie geteilt werden. Verkündigen ist eine Praxis religiöser Subjekte, die von Priestern und Propheten, amtlichen und charismatischen Predigern ausgeübt werden kann. Verkündigen macht die Inhalte einer Religion bekannt und ist insofern informativ. Dies geschieht freilich nicht im Sinne neutraler Information, sondern einer engagierten Mitteilung, die darauf aus ist, daß sich die Adressaten das Mitgeteilte zu eigen machen. Verkündigen ist zugleich immer kommunikativ und situativ" (ebd., 160-161). Auch hier wird die Spannung zwischen objektivem und subjektivem Anteil erkennbar. Wenn diese Spannung nicht aufgegeben wird, können in den Texten des Synodalen Weges die scheinbaren Spannungen eingeordnet und ausgehalten werden.

\section{„4. Teilen}

Eine wichtige Form kommunikativ-religiöser Praxis ist schließlich das Teilen. Es ist dies ein allgemeinerer Terminus für das, was christlich gesehene - diakonische Praxis heißt. Eine wichtige Form kommunikativ-religiöser Praxis ist schließlich das Teilen. Es ist ein allgemeinerer Terminus für das, was - christlich gesehen - diakonische Praxis heißt. Eine solche Praxis der Nächstenliebe, des Mitleidens, des Erbarmens, der Solidarität ist für alle Religionen zentral; sie gehört überall zum ethischen, kommunikativen Kern der Religion. Die Praxis des Teilens schafft, stärkt und transformiert in ganz besonderer Weise Gemeinschaft“" (ebd., 162) Vielleicht braucht es hier ergänzende Ideen für den Synodalen Weg.

Lamb betont - und trifft damit den Kern unserer Fragestellung mit Arens, dass es unmöglich sei, ,die Dichotomie von Lehren und Handeln, Lehrgehalt und Text, Bezeugen der Wahrheit und Bekennen in Wort und Tat aufrecht zu erhalten, sobald man die konstitutive Bedeutung und Wahrheit der kommunikativen Praxis des Bekennens des Glaubens ernst genommen hat “19. Für das Gelingen des Synodalen Weges wird es also darauf ankommen,

\footnotetext{
${ }^{19}$ Matthew L. Lamb, „Kommunikative Praxis, die Offenheit der Geschichte und die Dialektik von Gemeinschaft und Herrschaft", in Anerkennung der Anderen eine theologische Grunddimension interkultureller Kommunikation, 167-192 unter Verweis auf Arens, Bezeugen, 353-404.
} 
Wahrheit im kommunikativen Zusammenhang und mit biblischem und historischem Rückgriff dynamisch zu verstehen und die Anerkennung der Andren, auch ihrer unterscheidbaren Positionen und Praxis wertzuschätzen.

\section{BIBLIOGRAPHIE}

Arens, Edmund. Bezeugen und Bekennen elementare Handlungen des Glaubens. Düsseldorf: Patmos, 1989.

Arens, Edmund. „Konturen einer praktischen Religionstheorie“. In Anerkennung der Anderen eine theologische Grunddimension interkultureller Kommunikation [Helmut Peukert zum 60sten Geburtstag], ed. Edmund Arens, 138-166. Freiburg: Herder, 1995.

Bahr, Petra. „Religion und Aufklärung“. Rheinischen Merkur, 9. März 2006. Accessed: Januar 4, 2021, https://www.ekd.de/060313_bahr_religion_aufklaerung.htm.

Davie, Grace. Religion in Britain since 1945: Believing without belonging. Oxford: Wiley-Blackwell, 1994.

Ignatius von Loyola. Geistliche Übungen: Übertragung aus dem spanischen Urtext, Erklärung der 20 Anweisungen von Adolf Haas. Freiburg: Herder, 1967.

Knauer, Peter. Den Verstand abgeben? Ignatius von Loyola über Sehen und Glauben (2010). Accessed: December 31, 2020, http://peter-knauer.de/ignatius1.html.

Lamb, Matthew L. „Kommunikative Praxis, die Offenheit der Geschichte und die Dialektik von Gemeinschaft und Herrschaft“". In Anerkennung der Anderen eine theologische Grunddimension interkultureller Kommunikation [Helmut Peukert zum 60sten Geburtstag], ed. Edmund Arens, 167-192. Freiburg: Herder, 1995.

Loffeld, Jan. Der nicht notwendige Gott: Die Erlösungsdimension als Krise und Kairos des Christentums inmitten seines säkularen Relevanzverlustes. Würzburg: Echter Verlag, 2020.

Luhmann, Niklas. Die Religion der Gesellschaft. Frankfurt am Main: Suhrkamp, 2000.

Neuner, Peter. Der lange Schatten des I. Vatikanums wie das Konzil die Kirche noch heute blockiert. Freiburg-Basel-Wien: Herder, 2019.

Satzung des Synodalen Weges. Accessed: Januar 4, 2021, https://www.synodalerweg.de/filead min/Synodalerweg/Dokumente_Reden_Beitraege/Satzung-des-Synodalen-Weges.pdf.

Suess, Paolo. „Über die Unfähigkeit der Einen, sich der Andern zu erinnern“. In Anerkennung der Anderen eine theologische Grunddimension interkultureller Kommunikation; [Helmut Peukert zum 60sten Geburtstag], ed. Edmund Arens, 64-94. Freiburg: Herder, 1995.

Taylor, Charles. Ein säkulares Zeitalter. Frankfurt am Main: Suhrkamp, 2009.

Wolf, Hubert. Der Unfehlbare Pius IX. und die Erfindung des Katholizismus im 19. Jahrhundert: Biographie. München: C.H. Beck, 2020. 


\title{
AUF DER SUCHE NACH DER WAHRHEIT GOTTES - NEUES SELBSTVERSTÄNDNIS DER KIRCHE
}

\author{
Zusammenfassung
}

Die Struktur der katholischen Kirche befindet sich nach dem großen Glaubwürdigkeitsverlust ihrer Autorität in einem zentralen Transformationsprozess. Der Synodale Weg in Deutschland ist ein Versuch, die Anerkennung der Anderen neu zuzulassen von einem objektivistischen zu einem kommunikativen Wahrheitsverständnis voranzuschreiten. Bei diesem Prozess wird es nicht zuerst um konkrete Reformvorschläge gehen, die mehrheitsfähig sind. Vielmehr stehen die Frage nach der Einheit des Glaubens und nach den hermeneutischen Prozessen der Glaubensformulierung im Brennpunkt.

Schlüsselwörter: Wahrheit; Synodalität; Alterität; Glaubwürdigkeit; Aufklärung; Säkularisierung; Autorität; Bischofsamt.

W POSZUKIWANIU PRAWDY BOŻEJ

- NOWEGO SAMOZROZUMIENIA KOŚCIOŁA

\section{Streszczenie}

Po wielkiej utracie wiarygodności swojego autorytetu, struktura Kościoła katolickiego znajduje się w centralnym procesie przemian. Droga Synodalna w Niemczech jest próbą umożliwienia rozpoznania innym przejścia od obiektywistycznego do komunikatywnego rozumienia prawdy. $\mathrm{W}$ procesie tym nie chodzi w pierwszej kolejności o konkretne propozycje reform, które są do przyjęcia przez większość. W centrum zainteresowania pojawia się raczej pytanie o jedność wiary $\mathrm{i}$ hermeneutyczne procesy formułowania wiary.

Słowa kluczowe: prawda; synodalność; odmienność; wiarygodność; oświecenie; sekularyzacja; autorytet; episkopat. 